TRANSACTIONS OF THE

AMERICAN MATHEMATICAL SOCIETY

Volume 357, Number 6, Pages 2235-2251

S 0002-9947(04)03518-4

Article electronically published on April 27, 2004

\title{
ANOSOV AUTOMORPHISMS ON COMPACT NILMANIFOLDS ASSOCIATED WITH GRAPHS
}

\author{
S. G. DANI AND MEERA G. MAINKAR
}

\begin{abstract}
We associate with each graph $(S, E)$ a 2-step simply connected nilpotent Lie group $N$ and a lattice $\Gamma$ in $N$. We determine the group of Lie automorphisms of $N$ and apply the result to describe a necessary and sufficient condition, in terms of the graph, for the compact nilmanifold $N / \Gamma$ to admit an Anosov automorphism. Using the criterion we obtain new examples of compact nilmanifolds admitting Anosov automorphisms, and conclude that for every $n \geq 17$ there exist a $n$-dimensional 2-step simply connected nilpotent Lie group $N$ which is indecomposable (not a direct product of lower dimensional nilpotent Lie groups), and a lattice $\Gamma$ in $N$ such that $N / \Gamma$ admits an Anosov automorphism; we give also a lower bound on the number of mutually nonisomorphic Lie groups $N$ of a given dimension, satisfying the condition. Necessary and sufficient conditions are also described for a compact nilmanifold as above to admit ergodic automorphisms.
\end{abstract}

\section{INTRODUCTION}

Let $N$ be a simply connected nilpotent Lie group and $\Gamma$ be a lattice in $N$; namely $\Gamma$ is a discrete subgroup such that $N / \Gamma$ is compact. If $\tau$ is a hyperbolic automorphism of $N$ such that $\tau(\Gamma)=\Gamma$ then we get a diffeomorphism $\bar{\tau}$ of $N / \Gamma$, defined by $\bar{\tau}(x \Gamma)=\tau(x) \Gamma$ for all $x \in N$, which is an Anosov diffeomorphism of the compact nilmanifold $N / \Gamma$. Anosov diffeomorphisms arising in this way are called Anosov automorphisms. All known examples of Anosov diffeomorphisms are on nilmanifolds or on manifolds finitely covered by them. Also by a result of A. Manning 7] all Anosov diffeomorphisms on nilmanifolds are topologically conjugate to Anosov automorphisms. This raises the question as to which compact nilmanifolds admit Anosov automorphisms. Indeed it is easy to see that not all of them do. A first example of such a manifold other than a torus was described by S. Smale [11] (the example is attributed to A. Borel). Since then there have been several papers studying the class of compact nilmanifolds admitting Anosov automorphisms; see [1, [3], [4], 6] and the references cited in the last two papers.

In this paper we associate a compact nilmanifold with each graph, and describe necessary and sufficient conditions, in terms of the graph, for the manifold to admit Anosov automorphisms. This gives, in particular, new examples of compact nilmanifolds with Anosov automorphisms.

Received by the editors February 28, 2003 and, in revised form, July 16, 2003.

2000 Mathematics Subject Classification. Primary 22E25, 58F15; Secondary 22D40, 22D45, $05 \mathrm{C} 99$

The second-named author gratefully acknowledges partial support from the TIFR Alumni Association Scholarship of the TIFR Endowment Fund. 
Let $(S, E)$ be a finite graph, where $S$ is the set of vertices and $E$ the set of edges; equivalently $E$ is a collection of unordered pairs of distinct vertices; the unordered pairs will be written in the form $\alpha \beta$, where $\alpha, \beta \in S$. Let $V$ be a vector space with $S$ as a basis. Let $\wedge^{2} V$ be the second exterior power of $V$ and $W$ the subspace of $\wedge^{2} V$ spanned by $\{\alpha \wedge \beta: \alpha, \beta \in S, \alpha \beta \in E\}$. Let $\mathcal{N}=V \oplus W$. Stipulating the conditions that for any $\alpha, \beta \in S,[\alpha, \beta]=\alpha \wedge \beta$ if $\alpha \beta \in E$ and 0 otherwise, and $[v, w]=0$ for all $v \in V$ and $w \in W$ determines a unique Lie algebra structure on $\mathcal{N}$. Furthermore it can be seen that $\mathcal{N}$ is a 2-step nilpotent Lie algebra. Let $N$ be the simply connected Lie group with Lie algebra $\mathcal{N}$. We note that $N$ can be realised as $\mathcal{N}$ with the multiplication defined by

$$
\left(v_{1}, w_{1}\right) \cdot\left(v_{2}, w_{2}\right)=\left(v_{1}+v_{2}, w_{1}+w_{2}+\frac{1}{2}\left[v_{1}, v_{2}\right]\right),
$$

for all $v_{1}, v_{2} \in V$ and $w_{1}, w_{2} \in W$ (here + denotes the addition in $\mathcal{N}$ ).

Let $\Gamma$ be the subgroup of $N$ generated by $S$. It can be seen that when $N$ is identified with $\mathcal{N}$ as above, as a subset $\Gamma$ is contained in the (additive) subgroup of $\mathcal{N}$ generated by $S \cup\left\{\frac{1}{2}(\alpha \wedge \beta): \alpha \beta \in E\right\}$. In particular, $\Gamma$ is discrete. It can be further verified that $N / \Gamma$ is compact. Thus $\Gamma$ is a lattice in $N$, and $N / \Gamma$ is a compact nilmanifold; we call it the compact nilmanifold associated with $(S, E)$.

To describe the condition for $N / \Gamma$ as above to admit an Anosov automorphism we introduce the following definition. A subset $S^{\prime}$ of $S$ is said to be coherent if for all $\alpha, \beta \in S^{\prime}$ the following holds: if $\gamma \in S$ is such that $\alpha \gamma \in E$, then either $\gamma=\beta$ or $\beta \gamma \in E$.

Theorem 1.1. Let $(S, E)$ be a finite graph and let $N / \Gamma$ be the compact nilmanifold associated with $(S, E)$. Then $N / \Gamma$ admits an Anosov automorphism if and only if for every $\alpha \in S$ there exists a coherent subset $S^{\prime}$ of $S$ such that $\alpha \in S^{\prime}$ and one of the following conditions holds:

i) $S^{\prime}$ has at least 3 elements; or

ii) $S^{\prime}=\{\alpha, \beta\}$ for some $\beta \neq \alpha$ such that $\alpha \beta$ is not an edge.

As we shall see the set $S$ can be partitioned into maximal coherent subsets which we call the coherent components of $S$; conditions (i) and (ii) in the theorem are satisfied for some $S^{\prime}$ containing $\alpha$ if and only if they are satisfied for the coherent component of $\alpha$. We determine the coherent components for various classes of examples of graphs. Applying the theorem we then get new examples of compact nilmanifolds with and without Anosov automorphisms; see Corollary 6.4

We say that a simply connected nilpotent Lie group $N$ is indecomposable if it cannot be expressed as a direct product of two proper closed connected normal subgroups; that is, if $N_{1}$ and $N_{2}$ are two closed connected normal subgroups such that $N_{1} \cap N_{2}$ is trivial and $N=N_{1} N_{2}$, then either $N_{1}=N$ or $N_{2}=N$. Given a simply connected nilpotent Lie group $N$ and a lattice $\Gamma$ in $N, N$ is said to be $\Gamma$-indecomposable if it cannot be expressed as a direct product of two proper closed connected normal subgroups $N_{1}$ and $N_{2}$ such that $N_{1} \cap \Gamma$ and $N_{2} \cap \Gamma$ are lattices in $N_{1}$ and $N_{2}$ respectively. Clearly, if $N$ is indecomposable, then it is $\Gamma$ indecomposable for any lattice $\Gamma$ in it. Since given two compact nilmanifolds $N_{1} / \Gamma_{1}$ and $N_{2} / \Gamma_{2}$ admitting Anosov automorphisms the cartesian product $\left(N_{1} \times N_{2}\right) /$ $\left(\Gamma_{1} \times \Gamma_{2}\right)$ also admits Anosov automorphisms, the classification of compact nilmanifolds $N / \Gamma$ admitting Anosov automorphisms reduces to identifying all such $N / \Gamma$ 
where $N$ is $\Gamma$-indecomposable. From our results on existence of Anosov automorphisms we deduce, in particular, the following:

Corollary 1.2. For every integer $n \geq 17$ there exists an indecomposable simply connected 2-step nilpotent Lie group $N$ of dimension $n$, admitting a lattice $\Gamma$ such that $N / \Gamma$ has an Anosov automorphism. Furthermore, if $\nu(n)$ is the number of mutually nonisomorphic Lie groups satisfying this condition, then $\nu(n) \geq$ $\frac{1}{12}(n-3 \sqrt{2 n}-17)$.

We show also that if for some $\alpha \in S$ there is no coherent subset of $S$ containing at least two elements, namely if the coherent component of $\alpha$ consists only of itself, then $N / \Gamma$ does not admit any ergodic automorphism; that is, for any Lie automorphism $\tau$ of $N$ such that $\tau(\Gamma)=\Gamma$, the induced diffeomorphism on $N / \Gamma$ is not ergodic with respect to the $N$-invariant measure on $N / \Gamma$. It will be seen that this happens for several examples of graphs; see Corollary 6.4 An example with this property was given earlier in [5].

In the following sections we first obtain a description of the automorphism group of the simply connected nilpotent Lie group $N$ as above (or equivalently of the Lie algebra $\mathcal{N}$ ) associated with $(S, E)$; see Theorem 4.2. In particular, we get a description of the semisimple Levi component of the automorphism group, in terms of the coherent components of $(S, E)$; see Remark 4.4 These results may also be of independent interest. The description of the automorphism group is then applied, in 96 , together with standard properties of Anosov automorphisms to conclude Theorem 1.1.

\section{Preliminaries}

Let $\mathcal{N}$ be a 2 -step nilpotent Lie algebra; that is, $[\mathcal{N},[\mathcal{N}, \mathcal{N}]]=0$. Let $\mathcal{N}$ be expressed as $\mathcal{N}=V \oplus[\mathcal{N}, \mathcal{N}]$, where $V$ is a vector subspace complementary to $[\mathcal{N}, \mathcal{N}]$. We denote by $\operatorname{Aut}(\mathcal{N})$ the group of Lie algebra automorphisms of $\mathcal{N}$. We now obtain a decomposition of $\operatorname{Aut}(\mathcal{N})$ corresponding to the decomposition of $\mathcal{N}$ as above.

For any two vector spaces $V_{1}$ and $V_{2}$ we denote by $\operatorname{Hom}\left(V_{1}, V_{2}\right)$ the vector space of all linear maps of $V_{1}$ into $V_{2}$. Let $\pi: \mathcal{N} \rightarrow V$ be the canonical quotient linear map with respect to the decomposition of $\mathcal{N}$ as above. To each $\theta \in \operatorname{Hom}(V,[\mathcal{N}, \mathcal{N}])$ we associate a map $\tau_{\theta}: \mathcal{N} \rightarrow \mathcal{N}$ defined by $\tau_{\theta}(\xi)=\xi+\theta(\pi(\xi))$ for all $\xi \in \mathcal{N}$. Clearly $\tau_{\theta}$ is a linear automorphism and since for $\xi, \eta \in \mathcal{N}, \tau_{\theta}([\xi, \eta])=[\xi, \eta]+$ $\theta(\pi([\xi, \eta]))=[\xi, \eta]$, which also equals $[\xi+\theta(\pi(\xi)), \eta+\theta(\pi(\eta))]$, it follows that $\tau_{\theta}$ is a Lie algebra automorphism of $\mathcal{N}$. Any automorphism arising in this way, from some $\theta \in \operatorname{Hom}(V,[\mathcal{N}, \mathcal{N}])$, is called a shear automorphism.

Let $U$ be the subgroup of $\operatorname{Aut}(\mathcal{N})$ consisting of all shear automorphisms. It is easy to see that $U$ is a normal subgroup of $\operatorname{Aut}(\mathcal{N})$. Let $T=\{\tau \in \operatorname{Aut}(\mathcal{N}): \tau(V)=$ $V\}$. We note that $U \cap T$ is trivial; if $\theta \in \operatorname{Hom}(V,[\mathcal{N}, \mathcal{N}])$ is such that $\tau_{\theta} \in T$, then $\theta(\pi(\xi))=0$ for all $\xi \in \mathcal{N}$, which shows that $\tau_{\theta}$ is the identity automorphism. Next, we prove the following.

Proposition 2.1. Aut $(\mathcal{N})$ is the semidirect product of the subgroups $T$ and $U$.

Proof. Let $\tau \in \operatorname{Aut}(\mathcal{N})$. Then there exist uniquely defined linear transformations $\phi: V \rightarrow V$ and $\psi: V \rightarrow[\mathcal{N}, \mathcal{N}]$ such that $\tau(v)=\phi(v)+\psi(v)$ for all $v \in V$. Since $[\mathcal{N}, \mathcal{N}]$ is $\tau$-invariant and $\tau$ is invertible it follows that $\phi$ is invertible. Now let 
$\theta \in \operatorname{Hom}(V,[\mathcal{N}, \mathcal{N}])$ be the map defined by $\theta(v)=\psi\left(\phi^{-1}(v)\right)$ for all $v \in V$. Then for any $v \in V$ we have $\tau_{\theta}^{-1}(\tau(v))=\tau_{\theta}^{-1}(\phi(v)+\psi(v))=\phi(v)+\psi(v)-\theta(\phi(v))=$ $\phi(v)$, since $\theta(\phi(v))=\psi(v)$. This shows that $\tau_{\theta}^{-1}(\tau(v)) \in V$ for all $V$. Therefore, $\tau_{\theta}^{-1} \tau \in T$, and hence $\tau \in U T$. This proves the proposition.

Let $G$ be the subgroup of $G L(V)$ consisting of the restrictions $\tau \mid V, \tau \in T$. We note that $V$ generates $\mathcal{N}$ as a Lie algebra, and hence $\tau \mapsto \tau \mid V$ is injective. Therefore, by Proposition 2.1. $\operatorname{Aut}(\mathcal{N})$ is a semidirect product of $G$ and $\operatorname{Hom}(V,[\mathcal{N}, \mathcal{N}])$. Hence to understand the automorphism group $\operatorname{Aut}(\mathcal{N})$ it suffices to determine $G$. It may be observed that $T$ is a Lie subgroup of $\operatorname{Aut}(\mathcal{N})$, as it is a closed subgroup, and since the restriction homomorphism is continuous it follows that $G$ is a Lie subgroup of $G L(V)$.

We note also that $V$ as above may be identified with $\mathcal{N} /[\mathcal{N}, \mathcal{N}]$, via the correspondence $v \leftrightarrow v+[\mathcal{N}, \mathcal{N}]$, and hence $G$ corresponds to a Lie subgroup of $G L(\mathcal{N} /[\mathcal{N}, \mathcal{N}])$. It is easy to see that the subgroup of $G L(\mathcal{N} /[\mathcal{N}, \mathcal{N}])$ defined in this way is independent of the choice of the subspace $V$ complementing $[\mathcal{N}, \mathcal{N}]$ in $\mathcal{N}$. In the sequel it would, however, be convenient to realise it as a subgroup of a specific complement $V$, rather than as a subgroup of $G L(\mathcal{N} /[\mathcal{N}, \mathcal{N}])$.

Now let $(S, E)$ be a finite graph and let $\mathcal{N}$ be the Lie algebra associated with it, as described in the introduction. We recall that $\mathcal{N}=V \oplus W$, where $V$ is a vector space with $S$ as a basis and $W$ is a subspace of $\wedge^{2} V$ spanned by $\{\alpha \wedge \beta: \alpha \beta \in E\}$, and the Lie bracket operation $[]:, \mathcal{N} \times \mathcal{N} \rightarrow \mathcal{N}$ is defined to be the unique bilinear map satisfying the following conditions:

i) for $\alpha, \beta \in S,[\alpha, \beta]=\alpha \wedge \beta$ if $\alpha \beta \in E$ and 0 otherwise;

ii) $[\alpha, \beta \wedge \gamma]=0$ for all $\alpha, \beta, \gamma \in S$;

iii) $[\alpha \wedge \beta, \gamma \wedge \delta]=0$ for all $\alpha, \beta, \gamma, \delta \in S$.

We note that $[\mathcal{N}, \mathcal{N}]=W$, which is contained in the center of $\mathcal{N}$, so $\mathcal{N}$ is a 2-step nilpotent Lie algebra; $[\mathcal{N}, \mathcal{N}]$ coincides with the center if the graph is connected.

We recall that $V \oplus \wedge^{2} V$ is the free 2-step nilpotent Lie algebra based on $V$, with the bracket operation determined by the relations $[u, v]=u \wedge v$ for all $u, v \in V$, and $[v, w]=0$ for all $v \in V$ and $w \in \wedge^{2} V$ (see [1], for instance). The Lie algebra $\mathcal{N}$ as above is the quotient of $V \oplus \wedge^{2} V$ by the subspace of $\wedge^{2} V$ spanned by $\alpha \wedge \beta$ such that $\alpha \beta$ is not an edge; this subspace is a Lie ideal in $V \oplus \wedge^{2} V$, since $\wedge^{2} V$ is the center. On the other hand, the free 2-step nilpotent Lie algebra is the Lie algebra associated to the complete graph on $S$, where $S$ is a basis of $V$.

We conclude this section with the following characterisation of nilpotent Lie algebras associated with graphs.

Proposition 2.2. Let $\mathcal{N}$ be a 2-step nilpotent Lie algebra expressed as $\mathcal{N}=V \oplus$ $[\mathcal{N}, \mathcal{N}]$, where $V$ is a vector subspace complementary to $[\mathcal{N}, \mathcal{N}]$. Let $G$ be the subgroup of $G L(V)$ consisting of restrictions of Lie automorphisms of $\mathcal{N}$ leaving $V$ invariant. Let $S$ be a basis of $V$, and $D$ be the subgroup of $G L(V)$ consisting of all elements that are represented by diagonal matrices, with respect to $S$. Then $G$ contains $D$ if and only if $\mathcal{N}$ is (up to Lie algebra isomorphism) the Lie algebra associated with a graph $(S, E)$ with $S$ as above.

Proof. It is straightforward to verify that if $\mathcal{N}$ is the Lie algebra associated with a graph $(S, E)$ on the set $S$, then $D$ is contained in $G$. Conversely, suppose that $G$ contains $D$. Let $\left\{\lambda_{\alpha}\right\}_{\alpha \in S}$ be such that for $\xi, \eta, \phi, \psi \in S, \lambda_{\xi} \lambda_{\eta} \neq \lambda_{\phi} \lambda_{\psi}$ unless $\{\xi, \eta\}=\{\phi, \psi\}$. Let $d \in D$ be the transformation defined by $d(\alpha)=\lambda_{\alpha} \alpha$ for all 
$\alpha \in S$. Then $d \in G$ and hence there exists $\tau \in \operatorname{Aut}(\mathcal{N})$ such that $\tau(\alpha)=\lambda_{\alpha} \alpha$ for all $\alpha \in S$. Then for any $\alpha, \beta \in S,[\alpha, \beta]$ is either 0 or an eigenvector of $\tau$ with $\lambda_{\alpha} \lambda_{\beta}$ as the corresponding eigenvalue. By the choice of $d$ these eigenvalues are distinct and the corresponding eigenvectors are linearly independent. Let $E$ be an edge set on $S$ defined by $\alpha \beta \in E$ if $[\alpha, \beta] \neq 0$. It is then easy to see, using the above observation, that $\mathcal{N}$ is Lie isomorphic to the Lie algebra associated with $(S, E)$.

There exist examples of 2-step nilpotent Lie algebras $\mathcal{N}$ such that the subgroup $G$ of $G L(\mathcal{N} /[\mathcal{N}, \mathcal{N}])$ as above does not contain all diagonal transformations (with respect to any basis); this holds, for instance, for the Lie algebra of the Lie group described in $\S 3$ of $[5$, but one can also construct substantially simpler examples. It follows, in particular, from Proposition 2.2, that not all Lie algebras are associated with graphs in our sense.

\section{Lie subalgebras of $\operatorname{End}(V)$}

In this section we digress to describe Lie subalgebras of $\operatorname{End}(V)$ that contain all "diagonal" transformations. Let $V$ be a finite-dimensional vector space with a basis $S$ and let $\mathcal{D}$ be the Lie subalgebra of $\operatorname{End}(V)$ consisting of all endomorphisms that are represented by diagonal matrices with respect to the basis $S$.

We say that a subset $\Theta$ of $S \times S$ is transitive if for any $\alpha, \beta, \gamma \in S$ such that $\alpha \neq \gamma$ and $(\alpha, \beta),(\beta, \gamma) \in \Theta$ we have $(\alpha, \gamma) \in \Theta$. To each transitive subset $\Theta$ of $S \times S$ we associate an equivalence relation $\sim$ on $S$ defined as follows: for $\alpha, \beta \in S$, $\alpha \sim \beta$ if either $\alpha=\beta$ or, both $(\alpha, \beta)$ and $(\beta, \alpha)$ are contained in $\Theta$; it is clear that $\sim$ is an equivalence relation.

For $\alpha, \beta \in S$ we denote by $E_{\alpha \beta}$ the element of $\operatorname{End}(V)$ such that $E_{\alpha \beta}(\beta)=\alpha$ and $E_{\alpha \beta}(\xi)=0$ for $\xi \in S, \xi \neq \beta$. We note that for any Lie subalgebra $\mathcal{G}$ of $\operatorname{End}(V)$ the set $\left\{(\alpha, \beta) \in S \times S: E_{\alpha \beta} \in \mathcal{G}\right\}$ is a transitive subset of $S \times S$.

The following result may be considered standard; a proof is included for the sake of completeness.

Proposition 3.1. Let $\mathcal{G}$ be a Lie subalgebra of $\operatorname{End}(V)$ containing $\mathcal{D}$. Let $\Theta=$ $\left\{(\alpha, \beta): \alpha, \beta \in S, E_{\alpha \beta} \in \mathcal{G}\right\}$. Let $\Lambda$ be the set of equivalence classes in $S$ with respect to the equivalence relation associated to the transitive subset $\Theta$, and for $\lambda \in \Lambda$ let $S_{\lambda}$ be the corresponding equivalence class. For each $\lambda$ let $V_{\lambda}$ be the subspace of $V$ spanned by $S_{\lambda}$. Then

$$
\mathcal{G}=\mathcal{D} \oplus \bigoplus_{(\alpha, \beta) \in \Theta} \mathbb{R} E_{\alpha \beta}=\mathcal{Q} \oplus \mathcal{M}
$$

where $\mathcal{Q}=\bigoplus_{\lambda \in \Lambda} \operatorname{End}\left(V_{\lambda}\right)$, viewed as a Lie subalgebra of $\operatorname{End}(V)$ via the canonical embedding, and $\mathcal{M}$ is a nilpotent Lie ideal in $\mathcal{G}$. Furthermore, the elements of $\Lambda$ can be enumerated as $\lambda_{1}, \ldots, \lambda_{k}$ so that $\bigoplus_{i \leq j} V_{\lambda_{i}}$ is $\xi$-invariant for every $\xi \in \mathcal{G}$ and $j=1, \ldots, k$.

Proof. Let $X \in \mathcal{G}$. Then it can be expressed as $X=X_{0}+\sum_{\alpha, \beta \in S, \alpha \neq \beta} \lambda_{\alpha \beta} E_{\alpha \beta}$, where $X_{0} \in \mathcal{D}$ and $\lambda_{\alpha \beta} \in \mathbb{R}$. Consider any $\alpha, \beta \in S$ such that $\alpha \neq \beta$ and $\lambda_{\alpha \beta} \neq 0$. As $\mathcal{G}$ is a Lie algebra containing $\mathcal{D}$ it follows that $\left[E_{\alpha \alpha},\left[E_{\beta \beta}, X\right]\right] \in \mathcal{G}$. This shows that $\lambda_{\beta \alpha} E_{\beta \alpha}+\lambda_{\alpha \beta} E_{\alpha \beta} \in \mathcal{G}$. Also $\left[E_{\beta \beta}, \lambda_{\beta \alpha} E_{\beta \alpha}+\lambda_{\alpha \beta} E_{\alpha \beta}\right]=\lambda_{\beta \alpha} E_{\beta \alpha}-\lambda_{\alpha \beta} E_{\alpha \beta} \in \mathcal{G}$. Therefore, $\lambda_{\alpha \beta} E_{\alpha \beta} \in \mathcal{G}$ and since $\lambda_{\alpha \beta} \neq 0$ we get that $E_{\alpha \beta} \in \mathcal{G}$. This proves the first equality asserted in the proposition. 
For each $\lambda \in \Lambda$ the subset $\left\{E_{\alpha \beta}: \alpha, \beta \in S_{\lambda}\right\}$ generates the Lie subalgebra $\operatorname{End}\left(V_{\lambda}\right)$. Since these subsets are contained in $\Theta$, it follows that the Lie algebra $\mathcal{Q}$ as defined in the statement of the proposition is contained in $\mathcal{G}$. The Lie algebra $\operatorname{End}(V)$ can be decomposed as $\mathcal{Q} \oplus \bigoplus_{\lambda, \mu \in \Lambda, \lambda \neq \mu} \operatorname{Hom}\left(V_{\mu}, V_{\lambda}\right)$. It can be seen that for each $\lambda, \mu, \lambda \neq \mu, \operatorname{Hom}\left(V_{\lambda}, V_{\mu}\right)$ is invariant under the restriction of the adjoint action of $\operatorname{End}(V)$ to $\mathcal{Q}$, and that the $\mathcal{Q}$-action on $\operatorname{Hom}\left(V_{\lambda}, V_{\mu}\right)$ is irreducible. Since $\mathcal{G}$ is a Lie subalgebra of $\operatorname{End}(V)$ containing $\mathcal{Q}$ it follows that $\mathcal{G}$ has the form $\mathcal{Q} \oplus$ $\bigoplus_{(\lambda, \mu) \in \Psi} \operatorname{Hom}\left(V_{\mu}, V_{\lambda}\right)$, for a some subset $\Psi$ of $(\Lambda \times \Lambda) \backslash \Delta$, where $\Delta$ denotes the diagonal $\{(\sigma, \sigma): \sigma \in \Lambda\}$. We note that $\Psi$ is uniquely defined. Furthermore, if $(\lambda, \mu) \in \Psi$ for some $\lambda, \mu \in \Lambda$, then $(\mu, \lambda) \notin \Psi$; otherwise the elements of $S_{\lambda}$ and $S_{\mu}$ would be equivalent under $\sim$, which is not the case since $\lambda$ and $\mu$ are distinct.

Let $\mathcal{M}=\bigoplus_{(\lambda, \mu) \in \Psi} \operatorname{Hom}\left(V_{\mu}, V_{\lambda}\right)$. Then we have $\mathcal{G}=\mathcal{Q} \oplus \mathcal{M}$. Furthermore, $\mathcal{M}$ is clearly a Lie subalgebra and it is invariant under the adjoint action of $\mathcal{Q}$, so it is a Lie ideal in $\mathcal{G}$.

Now consider the relation on $\Lambda$ defined by $\lambda \prec \mu$ if either $\lambda=\mu$ or $\operatorname{Hom}\left(V_{\mu}, V_{\lambda}\right)$ is contained in $\mathcal{G}$. Since $\mathcal{G}$ is a Lie algebra, the relation is transitive. Also, the above observation implies that it is antisymmetric. Thus it is a partial order. We can therefore write the elements of $\Lambda$ as $\lambda_{1}, \ldots, \lambda_{k}$ such that, for any $i=1, \ldots, k, \lambda_{i}$ is a minimal element of $\left\{\lambda_{i}, \ldots, \lambda_{k}\right\}$. Then for $i, j=1, \ldots, k, i \neq j, \operatorname{Hom}\left(V_{\lambda_{j}}, V_{\lambda_{i}}\right)$ can be contained in $\mathcal{G}$ only if $i<j$. Hence $\mathcal{M}$ is contained in $\bigoplus_{i<j} \operatorname{Hom}\left(V_{\lambda_{j}}, V_{\lambda_{i}}\right)$. This shows that $\mathcal{M}$ is nilpotent and also that $\bigoplus_{i<j} V_{\lambda_{i}}$ is invariant under every $\xi \in \mathcal{G}$, for all $j=1, \ldots, k$. This completes the proof of the proposition.

\section{Determination of $G$}

We now return to the graph $(S, E)$ and the Lie algebra $\mathcal{N}$ associated with it, as in $\$ 1$. Let $G$ be the group of automorphisms of $\mathcal{N}$ as in $\$ 2$. For any $\sigma \in S$ we define

$$
\Omega^{\prime}(\sigma)=\{\omega \in S: \sigma \omega \in E\} \text { and } \Omega(\sigma)=\Omega^{\prime}(\sigma) \cup\{\sigma\} .
$$

Proposition 4.1. Let I denote the identity transformation in $G L(V)$. Let $\alpha, \beta \in$ $S, \alpha \neq \beta$. Then the following conditions are equivalent:

i) $I+E_{\alpha \beta} \in G$;

ii) $I+t E_{\alpha \beta} \in G$ for all $t \in \mathbb{R}$;

iii) $\Omega^{\prime}(\alpha) \subset \Omega(\beta)$.

Proof. i) $\Longrightarrow$ iii) Let $\tau=I+E_{\alpha \beta} \in G$; thus $\tau(\gamma)=\gamma$ for $\gamma \neq \beta$, and $\tau(\beta)=\beta+\alpha$. Consider any $\gamma \notin \Omega(\beta)$. Then $[\beta, \gamma]=0$. As $\tau \in G$, it is the restriction of a Lie automorphism of $\mathcal{N}$, and hence we get that $[\tau(\beta), \tau(\gamma)]=0$. That is, $[\beta+\alpha, \gamma]=0$. As $[\beta, \gamma]=0$ this shows that $[\alpha, \gamma]=0$. Therefore, $\gamma \notin \Omega^{\prime}(\alpha)$, since if $\alpha \gamma \in E$, then $[\alpha, \gamma]=\alpha \wedge \gamma \neq 0$. This shows that $\Omega^{\prime}(\alpha) \subset \Omega(\beta)$, so (iii) holds.

iii) $\Longrightarrow$ ii) Suppose that $\Omega^{\prime}(\alpha) \subset \Omega(\beta)$. Fix any $t \neq 0$ and let $\tau=I+t E_{\alpha \beta} \in$ $G L(V)$. Let $\wedge^{2} \tau: \wedge^{2} V \rightarrow \wedge^{2} V$ be the second exterior power of $\tau$. Let $W^{\prime}$ be the subspace of $\wedge^{2} V$ spanned by the set of all $\xi \wedge \eta$ such that $\xi \eta$ is not an edge. We show that $W^{\prime}$ is $\wedge^{2} \tau$-invariant. Let $\xi, \eta \in S$ be such that $\xi \neq \eta$ and $\xi \eta$ is not an edge. If neither of $\xi$ and $\eta$ equals $\beta$, then $\wedge^{2} \tau(\xi \wedge \eta)=\xi \wedge \eta \in W^{\prime}$. Now suppose $\xi=\beta$. Then $\eta \neq \beta$ and hence $\wedge^{2} \tau(\xi \wedge \eta)=(\beta+t \alpha) \wedge \eta=\beta \wedge \eta+t \alpha \wedge \eta$. As $\beta=\xi$, $\eta \notin \Omega(\beta)$, and since $\Omega^{\prime}(\alpha) \subset \Omega(\beta)$, it follows that $\eta \notin \Omega^{\prime}(\alpha)$. Hence both $\beta \wedge \eta$ and $\alpha \wedge \eta$ are contained in $W^{\prime}$. This shows that $\wedge^{2} \tau(\xi \wedge \eta) \in W^{\prime}$. Similarly, we see that if $\eta=\beta$, then also $\wedge^{2} \tau(\xi \wedge \eta) \in W^{\prime}$. This shows that $W^{\prime}$ is $\wedge^{2} \tau$-invariant. 
It is straightforward to see that $\left(\tau \oplus \wedge^{2} \tau\right): V \oplus \wedge^{2} V \rightarrow V \oplus \wedge^{2} V$ is a Lie algebra automorphism of the free 2-step nilpotent Lie algebra $V \oplus \wedge^{2} V$. Since $\mathcal{N}$ is the quotient of $V \oplus \wedge^{2} V$ by $W^{\prime}$, and the latter is $\wedge^{2} \tau$-invariant, it follows that $\tau \oplus \wedge^{2} \tau$ factors to a Lie algebra automorphism of $\mathcal{N}$ (one can also verify directly that the quotient of $\tau \oplus \wedge^{2} \tau$ on $\mathcal{N}$ is a Lie algebra automorphism of $\mathcal{N}$ ). Clearly $\tau$ is the restriction of this Lie automorphism to $V$. Hence $\tau=I+t E_{\alpha \beta} \in G$, and so (ii) holds.

As (ii) trivially implies (i), this completes the proof of the proposition.

Now let $\mathcal{G}$ be the Lie subalgebra of $\operatorname{End}(V)$ corresponding to $G$. We define

$$
\Theta=\Theta(S, E)=\left\{(\alpha, \beta) \in S \times S: \alpha \neq \beta \text { and } \Omega^{\prime}(\alpha) \subset \Omega(\beta)\right\} .
$$

We note that for any $\alpha, \beta \in S, \alpha \neq \beta,\left\{I+t E_{\alpha \beta}\right\}$ is the one-parameter subgroup in $G L(V)$ corresponding to the element $E_{\alpha \beta}$ of the Lie algebra $\operatorname{End}(V)$. Hence by Proposition 4.1 we get that $E_{\alpha \beta} \in \mathcal{G}$ if and only if $(\alpha, \beta) \in \Theta$. (This shows, in particular, that $\Theta$ is a transitive subset, but this can also be verified directly.)

Let $\Lambda=\Lambda(S, E)$ be the set of all equivalence classes in $S$ with respect to the equivalence relation associated with $\Theta$. Also for each $\lambda \in \Lambda$ let $S_{\lambda}$ be the corresponding equivalence class and let $V_{\lambda}$ be the subspace of $V$ spanned by $S_{\lambda}$. We note that each $S_{\lambda}, \lambda \in \Lambda$, is a coherent subset of $S$ with respect to $E$ (see $\$ 1$ for definition) and that every coherent subset of $S$ is contained in $S_{\lambda}$ for some $\lambda \in \Lambda$; $S_{\lambda}, \lambda \in \Lambda$, will be called the coherent components of $S$ with respect to $E$, and the decomposition of $V$ as $\bigoplus_{\lambda \in \Lambda} V_{\lambda}$ will be called the decomposition corresponding to the partition into coherent components.

Since $G$ contains $D$, it follows that $\mathcal{G}$ contains the Lie subalgebra $\mathcal{D}$ of $\operatorname{End}(V)$ consisting of diagonal transformations, and hence by Proposition 3.1 we get that

$$
\mathcal{G}=\mathcal{D} \oplus \bigoplus_{(\alpha, \beta) \in \Theta} \mathbb{R} E_{\alpha \beta}=\left(\bigoplus_{\lambda \in \Lambda} \operatorname{End}\left(V_{\lambda}\right)\right) \oplus \mathcal{M}
$$

where $\mathcal{M}$ is a nilpotent Lie ideal in $\mathcal{G}$, and that $\Lambda$ can be written as $\left\{\lambda_{1}, \ldots, \lambda_{k}\right\}$ so that $\bigoplus_{i \leq j} V_{\lambda_{i}}$ is $\xi$-invariant for all $\xi \in \mathcal{G}$ and $j=1, \ldots, k$. Let $M$ be the connected Lie subgroup of $G$ corresponding to $\mathcal{M}$. Then $M$ is a nilpotent Lie subgroup. It consists of unipotent elements and hence it is a closed subgroup. As $\mathcal{M}$ is the unique maximal nilpotent Lie ideal in $\mathcal{G}$, it is invariant under all Lie automorphisms of $\mathcal{G}$, so $M$ is invariant under all Lie automorphisms of $G$. In particular, it is normal in $G$. Then the connected component of the identity in $G$, which we denote by $G^{0}$, is given by

$$
G^{0}=\left(\prod_{\lambda \in \Lambda} G L^{+}\left(V_{\lambda}\right)\right) \cdot M
$$

where, for each $\lambda \in \Lambda, G L^{+}\left(V_{\lambda}\right)$ denotes the subgroup of $G L\left(V_{\lambda}\right)$ consisting of all the elements with positive determinant (the product is viewed as a subgroup of $G L(V)$ via the canonical embedding), and furthermore, $\bigoplus_{i \leq j} V_{\lambda_{i}}$ is $G^{0}$-invariant for each $j=1, \ldots, k$, where $\lambda_{1}, \ldots, \lambda_{k}$ are as above.

We note that $G$ is not connected, since the negative scalar transformations are contained in $G$ but they do not belong to the identity component $G^{0}$. The subgroup $D G^{0}$ of $G$ is an algebraic subgroup of $G L(V)$, and it is Zariski-connected as an algebraic subgroup. We note that $D G^{0}=\left(\prod_{\lambda \in \Lambda} G L\left(V_{\lambda}\right)\right) \cdot M ; M$ is the unipotent radical of $D G^{0}$ and $\prod_{\lambda \in \Lambda} G L\left(V_{\lambda}\right)$ is a (reductive) Levi subgroup. 
The subgroup $G$ can be even larger than $D G^{0}$. We next describe a subgroup of $G$ which may, in general, not be contained in $D G^{0}$.

Let $\Sigma(S, E)$ be the group of symmetries of $(S, E)$, namely permutations $\sigma$ of $S$ such that for $\alpha, \beta \in S, \alpha \beta \in E$ if and only if $\sigma(\alpha) \sigma(\beta) \in E$. Every $\sigma \in \Sigma(S, E)$ extends uniquely to a nonsingular linear transformation of $V$ and the latter can be seen to belong to $G$. The elements of $G$ arising in this way form a (finite) subgroup which also we shall denote by $\Sigma(S, E)$, identifying each symmetry $\sigma$ of $S$ with its extension to $V$. This subgroup may, in general, not be contained in $G^{0}$ (see examples in 45 . However, since $\Sigma(S, E)$ is contained in $G$, it normalises $G^{0}$. Since $M$ is the unique maximal connected nilpotent normal Lie subgroup of $G^{0}$, it follows that $\Sigma(S, E)$ normalises $M$. It follows that the Lie subalgebra $\mathcal{M}$ of $\operatorname{End}(V)$ is invariant under the action of $\Sigma(S, E) \subset G L(V)$, by conjugation. Unlike the reductive Levi component, the unipotent radical $M$ seems to depend more intricately, in a heuristic sense, on the specific details of the graph, and in this respect it is useful to know that it is invariant under the symmetry group $\Sigma(S, E)$.

In the examples in $\$ 5$ we shall see instances when $\Sigma(S, E)$ is not contained in $D G^{0}$. It seems plausible that $\Sigma(S, E)$ and $D G^{0}$ always generate $G$, but we are unable to ascertain this at present.

The results on the automorphism group may be summed up as follows.

Theorem 4.2. Let $(S, E)$ be a finite graph, $\mathcal{N}$ be the 2-step nilpotent Lie algebra associated with $(S, E)$, and $V$ and $W$ be as in the definition of $\mathcal{N}$. Let $\Theta(S, E)$ be the subset of $S \times S$ defined as above, and let $V=\bigoplus_{\lambda \in \Lambda} V_{\lambda}$ be the decomposition of $V$ corresponding to the partition of $S$ into coherent components. Then the automorphism group $\operatorname{Aut}(\mathcal{N})$ is a semidirect product of the subgroup $U$ consisting of shear automorphisms, and the subgroup $T$ consisting of all automorphisms leaving $V$ invariant. Each $\tau \in T$ is uniquely determined by its restriction $\left.\tau\right|_{V}$ on $V$, and for the Lie subgroup $G=\left\{\left.\tau\right|_{V}: \tau \in T\right\}$ of $G L(V)$ the following conditions are satisfied:

i) The Lie subalgebra $\mathcal{G}$ of $\operatorname{End}(V)$ corresponding to $G$ is given by

$$
\mathcal{G}=\mathcal{D} \oplus \bigoplus_{(\alpha, \beta) \in \Theta(S, E)} \mathbb{R} E_{\alpha \beta}
$$

ii) $G^{0}$ can be expressed as $\left(\prod_{\lambda \in \Lambda} G L^{+}\left(V_{\lambda}\right)\right) \cdot M$, where $M$ is a closed connected nilpotent normal subgroup of $G$.

iii) $G$ contains $\Sigma(S, E)$ and, in particular, $G^{0}$ and $M$ are normalised by $\Sigma(S, E)$.

iv) The elements of $\Lambda$ can be arranged as $\lambda_{1}, \ldots, \lambda_{k}$ so that for all $j=1, \ldots, k$, $\bigoplus_{i \leq j} V_{\lambda_{i}}$ is invariant under the action of $G^{0}$.

Remark 4.3. As mentioned earlier the group $G$ as above is not connected. We note, however, that $T$ is an algebraic subgroup of $G L(\mathcal{N})$, so it has only finitely many connected components (see [2]), and hence $G$ has only finitely many connected components, the identity component being as given by the theorem. The first part of the theorem then implies also that $\operatorname{Aut}(\mathcal{N})$ has only finitely many connected components.

Remark 4.4. Let $(S, E)$ be a finite graph. Then, with the notation as above, the following holds:

i) Assertion (ii) in Theorem 4.2 shows, in particular, that $\left(\prod_{\lambda \in \Lambda} S L\left(V_{\lambda}\right)\right)$ is a semisimple Levi component of $G^{0}$. Thus $G^{0}$ is solvable if and only if all coherent 
components of $S$ with respect to $E$ are singleton sets; when $G^{0}$ is solvable, the connected component of the identity in $\operatorname{Aut}(\mathcal{N})$ is also solvable.

ii) $G^{0}$ is a reductive Lie group if and only if for any $\alpha, \beta \in S$ such that $\Omega^{\prime}(\alpha) \subset$ $\Omega(\beta)$ we have $\Omega^{\prime}(\beta) \subset \Omega(\alpha)$.

Remark 4.5. It may be noted that the graph $(S, E)$ is not assumed to be connected. When $(S, E)$ is not connected, the isolated points in $S$ (namely those from which there is no edge) form a single coherent component, and all other coherent components are precisely the coherent components of the connected components of $(S, E)$ other than isolated points.

Remark 4.6. Let $(S, E)$ be a finite graph and let $S_{\lambda}, \lambda \in \Lambda$ be the family of its coherent components. It is easy to see that for any $\lambda \in \Lambda$, if there exist $\alpha, \beta \in S_{\lambda}$ such that $\alpha \beta \in E$, then $\xi \eta \in E$ for all $\xi, \eta \in S_{\lambda}$; that is, the restriction of $(S, E)$ to $S_{\lambda}$ is either a complete graph (i.e., every pair of vertices is connected by an edge) or it is discrete (has no edges).

Remark 4.7. Now let $\mathcal{E}$ be the set of all unordered pairs $\lambda \mu$ with $\lambda, \mu \in \Lambda$, for which there exist $\alpha \in S_{\lambda}$ and $\beta \in S_{\mu}$ such that $\alpha \beta \in E$. We call $(\Lambda, \mathcal{E})$ the coherence class graph. Let $\Lambda_{0}$ be the subset of $\Lambda$ consisting of all $\lambda$ such that the restriction of $(S, E)$ to $S_{\lambda}$ is a complete graph. In view of Remark 4.6 (and the definition of coherent subsets) the graph $(\Lambda, \mathcal{E})$ and $\Lambda_{0}$ together determine the graph $(S, E)$; given $\alpha, \beta \in S$, with say $\alpha \in S_{\lambda}$ and $\beta \in S_{\mu}, \alpha \beta \in E$ if and only if either $\lambda=\mu \in \Lambda_{0}$ or $\lambda \neq \mu$ and $\lambda \mu \in \mathcal{E}$. We note also that given a graph $(\Lambda, \mathcal{E})$ in which no two distinct vertices are coherent to each other, a family of positive integers $\left\{m_{\lambda}\right\}_{\lambda \in \Lambda}$ and a subset $\Lambda_{0}$ of $\Lambda$ there exists a graph $(S, E)$ such that $(\Lambda, \mathcal{E})$ may be realised (up to equivalence of graphs) as the coherence class graph of $(S, E)$, for $\lambda \in \Lambda$ the corresponding coherent class $S_{\lambda}$ has cardinality $m_{\lambda}$, and the restriction of $(S, E)$ to $S_{\lambda}$ is complete or discrete according to whether $\lambda \in \Lambda_{0}$ or $\lambda \notin \Lambda_{0}$, respectively; furthermore, such a graph $(S, E)$ is unique.

\section{Some EXAMPLES OF GRAPHS}

In this section we discuss the set $\Theta(S, E)$ and the partition into coherent components, in respect to some classes of graphs (for the associated Lie algebra, Lie group, etc., we follow the notation as in the preceding sections).

1. Complete graphs: Let $(S, E)$ be a complete graph on a finite set. Then clearly $\Theta(S, E)=\{(\alpha, \beta): \alpha, \beta \in S, \alpha \neq \beta\}$; hence $S$ is a coherent set and $G=G L(V)$.

2. Chain graphs: Consider the "chain" graphs $(S, E)$, where $S=\{1,2, \ldots, n\}$, $n \geq 2$, and $E=\{k(k+1): k=1, \ldots, n-1\}$. Then we see that $\Theta(S, E)=$ $\{(1,2),(1,3),(n, n-2),(n, n-1)\}$. For $n=2, S$ is coherent, and hence $G=G L(V)$. For $n=3$ the coherent components are $\{1,3\}$ and $\{2\}$, and $G$ can be seen to be the parabolic subgroup of $G L(V)$ leaving invariant the subspace spanned by the vertices 1 and 3. For $n \geq 4$ all the coherent components are singleton sets; in this case $G^{0}$ is a solvable Lie group; furthermore, $\left[G^{0}, G^{0}\right]$ coincides with the nilradical $M$ and is commutative.

3. Cycles: Let $(S, E)$ be the cycle graph on $n$ vertices, where $n \geq 3$, namely $S=\{1,2, \ldots, n\}$ and $E=\{k(k+1): k=1, \ldots, n-1\} \cup\{n 1\}$. For $n=3,(S, E)$ is a complete graph, considered in 1 above. For $n=4, \Theta(S, E)=\{(i, j):|i-j|=2\}$ and hence the coherent components are $\{1,3\}$ and $\{2,4\}$. The group $G$ can be seen to be generated by $G L\left(V_{1}\right) \times G L\left(V_{2}\right)$ where $V_{1}$ and $V_{2}$ are the subspaces of $V$ 
spanned by the subsets $S_{1}=\{1,3\}$ and $S_{2}=\{2,4\}$, together with the permutation $\sigma=(1,2)(3,4)$ (product of the two transpositions).

For $n \geq 5, \Theta(S, E)$ can be seen to be empty; consequently, all coherent components are singletons. In this case $G$ can be seen to be generated by $D$ and the permutation $(1,2, \ldots, n)$ (since $G$ contains $D$ as a normal subgroup it follows that it is generated by $D$ and the permutations contained in it, and the latter can be seen to be contained in the subgroup generated by the $(1,2, \ldots, n))$.

4. Trees: A tree is a connected graph $(S, E)$ having no subgraph which is a cycle. In this case it can be seen that two end vertices (through which there is only one edge) belong to the same coherent component if they belong to the same final branch (namely if the other vertex of the edges is common); each of the interior vertices (other than end vertices) constitutes a coherent component by itself.

5. Complete bipartite graphs and "clump" graphs: Let $S$ be a disjoint union of two subsets $S_{1}$ and $S_{2}$, each containing at least two elements, and let $E$ consist of $\alpha \beta$ such that one of $\alpha$ and $\beta$ belongs to $S_{1}$ and the other belongs to $S_{2}$. This is called a bipartite graph. In this case $\Theta(S, E)=\left\{(\alpha, \beta): \alpha, \beta \in S_{1}\right\} \cup\left\{(\alpha, \beta): \alpha, \beta \in S_{2}\right\}$. A more general class of graphs of this kind may be defined as follows: Let $S$ be a set which is a disjoint union of subsets $S_{1}, S_{2}, \ldots, S_{k}$, where $k \geq 2$ and each $S_{i}$ has at least two elements. Let $\mathcal{E}$ be the edge set of a connected graph on the set $\{1, \ldots, k\}$. We define the set $E$ of edges on $S$ to consist of unordered pairs $\alpha \beta$ where $\alpha \in S_{i}$ and $\beta \in S_{j}$ for some $i, j \in\{1, \ldots, k\}$, such that $(i \neq j$ and $) i j \in \mathcal{E}$. (This reduces to the bipartite graph when $k=2$.) A graph $(S, E)$ arising in this way, we shall call a clump graph. In this case

$$
\Theta(S, E)=\bigcup_{i, j \in\{1, \ldots, k\}, \Omega^{\prime}(i) \subset \Omega^{\prime}(j)}\left\{(\alpha, \beta): \alpha \in S_{i}, \beta \in S_{j}\right\},
$$

where $\Omega^{\prime}(j)$ are as defined in $\$ 4$ for the graph $(\{1, \ldots, k\}, \mathcal{E})$. It follows that $S_{1}, \ldots, S_{k}$ are coherent subsets; the coherent component of $S$ containing $S_{i}, i=$ $1, \ldots, k$, is given by $\bigcup_{\Omega^{\prime}(j)=\Omega^{\prime}(i)} S_{j}$.

6. "Magnet" graphs: Let $S$ be a finite set and $C$ a subset of $S$ such that at least two elements of $S$ are not contained in $C$. Consider the graph $E$ on $S$ given by the condition that $\alpha \beta$ is an edge, for $\alpha, \beta \in S$ with $\alpha \neq \beta$ if at least one of $\alpha$ and $\beta$ is contained in $C$; we call $C$ the core of the graph. In this case it can be seen that the partition of $S$ into coherent components consists of the partition into $C$ and its complement in $S$.

7. "Complementary" graphs: Let $(S, E)$ be a finite graph and $(S, \tilde{E})$ the graph over the same set of vertices, defined by the condition that, for $\alpha, \beta \in S, \alpha \beta \in \tilde{E}$ if and only if $\alpha \beta$ is not an edge in $E$; namely the set of edges of $E$ and $\tilde{E}$ are complementary to each other; we call $(S, \tilde{E})$ the complementary graph of $(S, E)$. It is easy to see that the coherent components of $S$ with respect to $E$ and $\tilde{E}$ are the same.

\section{Anosov automorphisms and ergodic automorphisms of $N / \Gamma$}

We now discuss the implications of Theorem 4.2 for Anosov automorphisms and ergodic automorphisms of $N / \Gamma$, and prove Theorem 1.1 .

Let $(S, E)$ be a finite graph and $N / \Gamma$ be the compact nilmanifold associated with it, $N$ and $\Gamma$ being as defined in $\$ 1$ Let $\mathcal{N}$ be the Lie algebra of $N$ in the form described in $₫ 1$. Let $V$ and $W$ be as in the definition of $\mathcal{N}$, so $\mathcal{N}=V \oplus W$. 
Let $\Phi$ be the subgroup of $\mathcal{N}$ (with respect to addition) generated by the subset $S \cup\left\{\frac{1}{2}(\alpha \wedge \beta): \alpha, \beta \in S, \alpha \beta \in E\right\}$. When $N$ is realised as $\mathcal{N}$ with the multiplication as described in 1 the group of Lie automorphisms of $N$ is the same as $\operatorname{Aut}(\mathcal{N})$ (in fact, the identity map of $\mathcal{N}$ is the corresponding exponential map). Also, $\Phi$ is a subgroup of $N$ and $\Gamma$ is the smallest subgroup such that $\Gamma[\Phi, \Phi]=\Phi$. Hence $N / \Gamma$ admits an Anosov automorphism if and only if there exists $\tau \in \operatorname{Aut}(\mathcal{N})$ such that $\tau$ is a hyperbolic linear transformation of $\mathcal{N}$ and $\tau(\Phi)=\Phi$.

Lemma 6.1. Let $\Lambda$ be the set of coherent components and let $\lambda_{1}, \ldots, \lambda_{k}$ be an enumeration of $\Lambda$ such that assertion (iv) of Theorem 4.2 holds. For each $j=$ $1, \ldots, k$ let $\mathcal{N}_{j}=\left(\bigoplus_{i \leq j} V_{\lambda_{i}}\right) \oplus W$; also let $\mathcal{N}_{0}=W$. Let $\tau$ be a Lie automorphism of $\mathcal{N}$ contained in the connected component of the identity in $\operatorname{Aut}(\mathcal{N})$. Then each $\mathcal{N}_{j}$ is invariant under the action of $\tau$. If $\tau(\Phi)=\Phi$, then for all $j=1, \ldots, k$ the determinant of the action of $\tau$ on $\mathcal{N}_{j}$ is \pm 1 .

Proof. The connected component of the identity in $\operatorname{Aut}(\mathcal{N})$ is the semidirect product of the identity component of $T$ and $U$, where $T$ and $U$ are the subgroups as in Theorem 4.2 The first assertion in the lemma therefore follows immediately from assertion (iv) of that theorem. Now suppose that $\tau(\Phi)=\Phi$. Observe that for each $j=1, \ldots, k, \mathcal{N}_{j} \cap \Phi$ is a lattice in $\mathcal{N}_{j}$, and it is invariant under the action of $\tau$. This implies the second assertion.

Proof of Theorem 1.1. Suppose that for each $\alpha \in S$ there exists a coherent subset $S^{\prime}$ such that (i) or (ii) holds. Let $S_{\lambda}, \lambda \in \Lambda$ be the coherent components of $S$ with respect to $E$ (see 4 (4). As every coherent subset of $S$ is contained in a coherent component $S_{\lambda}$, for some $\lambda \in \Lambda$, it follows that for each $\lambda \in \Lambda$ either $\left|S_{\lambda}\right| \geq 3$, or $S_{\lambda}=\{\alpha, \beta\}$ for some $\alpha, \beta \in S$ such that $\alpha \beta$ is not an edge.

As before let $V_{\lambda}$ be the subspace of $V$ spanned by $S_{\lambda}$. Hence $V=\bigoplus_{\lambda \in \Lambda} V_{\lambda}$. For each $\lambda \in \Lambda$ let $\Phi_{\lambda}$ be the subgroup of $V_{\lambda}$ generated by $S_{\lambda}$. A transformation from $G L\left(V_{\lambda}\right)$ leaves invariant the subgroup $\Phi_{\lambda}$ if and only if the matrix representing it with respect to the basis $S_{\lambda}$ belongs to $G L\left(d_{\lambda}, \mathbb{Z}\right)$ (nonsingular integral matrices with determinant \pm 1 ), where $d_{\lambda}=\left|S_{\lambda}\right|$. Therefore, for every $\lambda$ such that $\left|S_{\lambda}\right| \geq 3$ there exists $g_{\lambda} \in G L\left(V_{\lambda}\right)$ such that $g_{\lambda}\left(\Phi_{\lambda}\right)=\Phi_{\lambda}$, and the eigenvalues of $g_{\lambda}$ as well as pairwise products of the eigenvalues are of absolute value different from 1; existence of such elements can be deduced from the theorem of Gopal Prasad and Raghunathan [9] using the fact that $S L(d, \mathbb{Z})$ is a lattice in $S L(d, \mathbb{R})$ (see [3] for an idea of the argument); alternatively explicit examples may be constructed in low dimensions $(3,4$ and 5$)$ and put together. For $\lambda$ such that $\left|S_{\lambda}\right|=2$ let $g_{\lambda}$ be any hyperbolic element in $S L\left(V_{\lambda}\right)$ such that $g_{\lambda}\left(\Phi_{\lambda}\right)=\Phi_{\lambda}$. We choose natural numbers $j_{\lambda}, \lambda \in \Lambda$, such that if $\lambda$ and $\mu$ are distinct elements of $\Lambda$ and $a$ and $b$ are eigenvalues of $g_{\lambda}$ and $g_{\mu}$ respectively then $\left|a^{j_{\lambda}} b^{j_{\mu}}\right| \neq 1$. Now let $g \in G L(V)$ be the element whose restriction to $V_{\lambda}$ is $g_{\lambda}^{j_{\lambda}}$, for each $\lambda \in \Lambda$.

By Theorem 4.2 the element $g$ as above is contained in the subgroup $G$ of $G L(V)$, in the notation of the last section, and hence there exists $\tau \in \operatorname{Aut}(\mathcal{N})$ such that $g$ is the restriction of $\tau$ to $V$. Since $g$ leaves invariant the subgroup of $V$ generated by $S$ it follows that $\tau(\Phi)=\Phi$. We show that $\tau$ is hyperbolic as a linear transformation. Suppose if possible that it has an eigenvalue, say $c$, of absolute value 1 . Since $g$ has no eigenvalues of absolute value 1 , it follows that $c$ must be an eigenvalue of the restriction of $\tau$ to $W$. Then there exists a nonzero $\tau$-invariant subspace $W^{\prime}$ of $W$ such that all eigenvalues of $\tau$ on $W^{\prime}$ are of absolute value 1 . We note that 
$W=[\mathcal{N}, \mathcal{N}]=[V, V]=\sum_{\lambda, \mu \in \Lambda}\left[V_{\lambda}, V_{\mu}\right]$. For any $\lambda, \mu \in \Lambda,\left[V_{\lambda}, V_{\mu}\right]$ is $\tau$-invariant. Furthermore, the absolute values of the eigenvalues of $\tau$ over $\left[V_{\lambda}, V_{\mu}\right]$ are products of absolute values of the eigenvalues of $\tau$ over $V_{\lambda}$ and $V_{\mu}$. The conditions in the choice of $g$ therefore imply that for $\lambda, \mu \in \Lambda$ the restriction of $\tau$ to $\left[V_{\lambda}, V_{\mu}\right]$ has an eigenvalue of absolute value 1 only if $\lambda=\mu$ and $\left|S_{\lambda}\right|=2$. This implies that $W^{\prime}$ is contained in $\sum_{\lambda \in \Lambda,\left|S_{\lambda}\right|=2}\left[V_{\lambda}, V_{\lambda}\right]$, as may be seen by considering the quotient of $W$ modulo the latter subspace. Since $W^{\prime}$ is nonzero, we get, in particular, that there exists $\lambda_{0} \in \Lambda$ such that $\left|S_{\lambda_{0}}\right|=2$ and $\left[V_{\lambda_{0}}, V_{\lambda_{0}}\right]$ is nonzero. Let $S_{\lambda_{0}}=\{\alpha, \beta\}$. Then $\left[V_{\lambda_{0}}, V_{\lambda_{0}}\right]$ is the subspace spanned by $[\alpha, \beta]$. Therefore, the preceding conclusion implies that $[\alpha, \beta] \neq 0$. But since $\{\alpha, \beta\}=S_{\lambda_{0}}$, by the condition in the hypothesis $\alpha \beta$ is not an edge, and hence $[\alpha, \beta]=0$. The contradiction shows that $\tau$ is hyperbolic. Since $\tau \in \operatorname{Aut}(\mathcal{N})$ and $\tau(\Phi)=\Phi$, as noted earlier this implies that $N / \Gamma$ admits an Anosov automorphism.

Now suppose conversely that $N / \Gamma$ admits an Anosov automorphism. Hence there exists $\tau \in \operatorname{Aut}(\mathcal{N})$ such that $\tau$ is a hyperbolic linear transformation of $\mathcal{N}$ and $\tau(\Phi)=\Phi$. Since $\operatorname{Aut}(\mathcal{N})$ has only finitely many connected components (see Remark (4.3) by replacing $\tau$ by a suitable power we may assume that $\tau$ is contained in the connected component of the identity in $\operatorname{Aut}(\mathcal{N})$. Let $\mathcal{N}_{j}, j=1, \ldots, k$, be defined as in Lemma 6.1. Then by the lemma, for each $j=1, \ldots, k, \mathcal{N}_{j}$ is $\tau$-invariant and the determinant of the restriction of $\tau$ to any $\mathcal{N}_{j}$ is \pm 1 .

Suppose first that there exists $\lambda \in \Lambda$, say $\lambda=\lambda_{j}$, such that $S_{\lambda}$ is a singleton set. Then $V_{\lambda}$ is one-dimensional. Since $\mathcal{N}_{j}=\mathcal{N}_{j-1} \oplus V_{\lambda}$ the one-dimensionality of $V_{\lambda}$ together with the above conclusion implies that $\tau$ has \pm 1 as an eigenvalue. This is a contradiction, since by assumption $\tau$ is hyperbolic. Hence each $S_{\lambda}$ has at least two elements.

Next suppose that there exists $\lambda \in \Lambda$ such that $S_{\lambda}=\{\alpha, \beta\}$, with $\alpha, \beta \in S$ and $\alpha \beta \in E$. Let $j, 1 \leq j \leq k$, be such that $\lambda=\lambda_{j}$ and consider the action of $\tau$ on $\mathcal{N}_{j} / \mathcal{N}_{j-1}$. The latter is two-dimensional and since $\tau$ is hyperbolic, it follows that the action has two distinct real eigenvalues, say $\theta_{1}$ and $\theta_{2}$. Furthermore, the above observation on the determinants implies that $\theta_{1} \theta_{2}= \pm 1$. Therefore, there exist $v_{1}, v_{2} \in V_{\lambda}$ such that $\tau\left(v_{i}\right)=\theta_{i} v_{i}+x_{i}$, where $x_{i} \in \mathcal{N}_{j-1}$, for $i=1,2$. We can write $v_{i}, i=1,2$, as $v_{i}=a_{i} \alpha+b_{i} \beta$ where $a_{i}, b_{i} \in \mathbb{R}$. Then $\left[v_{1}, v_{2}\right]=\delta[\alpha, \beta]$, where $\delta=\left(a_{1} b_{2}-a_{2} b_{1}\right)$. Since $\theta_{1}$ and $\theta_{2}$ are distinct, $v_{1}$ and $v_{2}$ are linearly independent and hence $\delta \neq 0$. Now

$$
\tau\left(\left[v_{1}, v_{2}\right]\right)=\left[\tau\left(v_{1}\right), \tau\left(v_{2}\right)\right]=\left[\theta_{1} v_{1}+x_{1}, \theta_{2} v_{2}+x_{2}\right]=\theta_{1} \theta_{2}\left[v_{1}, v_{2}\right]+x,
$$

where $x$ is an element of $\left[\mathcal{N}_{j}, \mathcal{N}_{j-1}\right]$. Therefore,

$$
\tau([\alpha, \beta])=\theta_{1} \theta_{2}[\alpha, \beta]+\delta^{-1} x=c[\alpha, \beta]+x^{\prime},
$$

where $c=\theta_{1} \theta_{2}= \pm 1$ and $x^{\prime}=\delta^{-1} x \in\left[\mathcal{N}_{j}, \mathcal{N}_{j-1}\right]$. We note that since $\alpha \beta \in E$, $[\alpha, \beta]=\alpha \wedge \beta$, and that it is not contained in $\left[\mathcal{N}_{j}, \mathcal{N}_{j-1}\right]$. This shows that the action of $\tau$ on $\left[\mathcal{N}_{j}, \mathcal{N}_{j}\right]$ has $c= \pm 1$ as an eigenvalue. This is a contradiction, since by assumption $\tau$ is hyperbolic. This shows that if for some $\lambda \in \Lambda, S_{\lambda}=\{\alpha, \beta\}$, with $\alpha \neq \beta$, then $\alpha \beta$ is not an edge. This completes the proof of the theorem.

Theorem 4.2 can be applied similarly, in fact in a simpler way, to get a characterisation of compact nilmanifolds admitting ergodic automorphisms. Let $(S, E)$ be a finite graph and let $N / \Gamma$ be the compact nilmanifold associated with it, as 
defined in 1 . We recall that an automorphism of $N / \Gamma$ is ergodic (with respect to the unique probability measure invariant under the action of $N$ by translations) if and only if its factor on $N /[N, N] \Gamma$ is ergodic (see [8]). When $N$ is realised as $\mathcal{N}$ as before, this means, together with the criterion for ergodicity of toral automorphisms, that the automorphism of $N / \Gamma$ defined by $\tau \in \operatorname{Aut}(\mathcal{N})$ such that $\tau(\Gamma)=\Gamma$ is ergodic if and only if the factor of $\tau$ on $V$ has no eigenvalue which is a root of unity. Therefore, from Theorem 4.2 we can deduce the following.

Theorem 6.2. Let $N / \Gamma$ be the compact nilmanifold associated with a finite graph $(S, E)$. Then $N / \Gamma$ admits an ergodic automorphism if and only if every $\alpha \in S$ is contained in a coherent subset $S^{\prime}$ containing at least two elements.

Proof. As before let $S_{\lambda}, \lambda \in \Lambda$, be the partition of $S$ into coherent components, and $V_{\lambda}$ be the subspace of $V$ spanned by $S_{\lambda}$. Suppose that every $S_{\lambda}$ has at least two elements; this is equivalent to the second condition in the theorem. Let $A$ denote the subgroup of $V$ generated by $S$. For each $\lambda$ let $g_{\lambda} \in S L\left(V_{\lambda}\right)$ be an element such that $g_{\lambda}\left(V_{\lambda} \cap A\right)=V_{\lambda} \cap A$ and $g_{\lambda}$ has no eigenvalue which is a root of unity; it is easy to see that such a choice may be made. Let $g \in G L(V)$ be the element whose restriction to $V_{\lambda}$ is $g_{\lambda}$, for each $\lambda$. By Theorem $4.2 g$ is the restriction of an (unique) automorphism of $\mathcal{N}$, say $\tau \in \operatorname{Aut}(\mathcal{N})$. Since $g(A)=A$, it follows that $\tau(\Gamma)=\Gamma$ and hence $\tau$ induces an automorphism, say $\bar{\tau}$, of $N / \Gamma$. By the criterion recalled above and the choice of $g$ it follows that $\bar{\tau}$ is ergodic. This proves the "if" part of the theorem.

Now suppose that $N / \Gamma$ admits an ergodic automorphism. Thus there exists $\tau \in \operatorname{Aut}(\mathcal{N})$ such that $\tau(\Phi)=\Phi$ and the factor of $\tau$ on $V$ has no eigenvalue which is a root of unity; here $\Phi$ is the subgroup of $\mathcal{N}$ coinciding with $\Gamma$ when $N$ is realised as $\mathcal{N}$. As $\operatorname{Aut}(\mathcal{N})$ has only finitely many connected components, by replacing $\tau$ by a suitable power we may assume that $\tau$ is contained in the identity component of $\operatorname{Aut}(\mathcal{N})$. Let $\mathcal{N}_{j}, j=1, \ldots, k$, be defined as in Lemma 6.1 hence for each $j$ the subspace $\mathcal{N}_{j}$ is $\tau$-invariant and the determinant of the restriction of $\tau$ to any $\mathcal{N}_{j}$ is \pm 1 . Now suppose that there exists $\lambda \in \Lambda$, say $\lambda=\lambda_{j}$ such that $S_{\lambda}$ is a singleton. Then $V_{\lambda}$ is one-dimensional. As $\mathcal{N}_{j}=\mathcal{N}_{j-1} \oplus V_{\lambda}$ the preceding conclusion implies that \pm 1 is an eigenvalue of the factor of $\tau$ on $V=\mathcal{N} / W$. But this contradicts the ergodicity condition. Therefore, every $S_{\lambda}$ has at least two elements.

Remark 6.3. Let $(S, E)$ be a connected graph, where $S$ is a set with at least 3 elements. Suppose that all coherent components have at least 2 elements. Then every $\alpha \in S$ is contained in a 3 -cycle or a 4 -cycle in $(S, E)$. This may be seen as follows: For $\xi, \eta \in S$ we write $\xi \sim \eta$ if they are coherent to each other, namely in the same coherent component. Let $\alpha \in S$ be given. As $S$ is connected, there exists $\beta \in S$ such that $\alpha \beta \in E$. Suppose first that $\alpha \sim \beta$. Since $(S, E)$ is connected, there exists $\gamma \in S \backslash\{\alpha, \beta\}$ such that either $\alpha \gamma \in E$ or $\beta \gamma \in E$. Since $\alpha \sim \beta$ it follows that $\alpha \beta, \beta \gamma, \gamma \alpha \in E$; in this case $\alpha$ is contained in a 3 -cycle. Now suppose that $\alpha$ and $\beta$ are not coherent to each other. As each coherent component has at least two elements, we can choose $\alpha^{\prime}, \beta^{\prime} \in S$ such that $\alpha^{\prime} \sim \alpha$ and $\beta^{\prime} \sim \beta$. As $\alpha$ and $\beta$ are not coherent to each other it follows that $\alpha^{\prime}, \beta^{\prime} \in S \backslash\{\alpha, \beta\}$ and $\alpha^{\prime} \neq \beta^{\prime}$. It is easy to see that then $\left\{\alpha, \beta, \alpha^{\prime}, \beta^{\prime}\right\}$ is a 4 -cycle. This proves the claim.

Together with the observations in the last section, Theorems 1.1 and 6.2 imply the following corollary. 
Corollary 6.4. Let $(S, E)$ be a finite connected graph and $N / \Gamma$ be the compact nilmanifold associated with it. Suppose that $S$ has at least 3 elements. Then we have the following:

i) if there exists $\alpha \in S$ which is not contained in a p-cycle where $p=3$ or 4 (e.g. if $(S, E)$ is chain graph or a tree graph, or a cycle graph on $n \geq 5$ vertices), then $N / \Gamma$ does not admit any ergodic automorphism;

ii) if $(S, E)$ is a complete graph or a clump graph, then $N / \Gamma$ admits an Anosov automorphism.

iii) if $(S, E)$ is a magnet graph, then $N / \Gamma$ admits an Anosov automorphism if and only the core has at least 3 elements.

We note that if $S$ is a set with two elements and $(S, E)$ is the (unique) connected chain graph on it, then $N$ is the 3-dimensional Heisenberg group, and in this case $N / \Gamma$ admits an ergodic automorphism but not an Anosov automorphism. The assertion in (i) for chain graphs over $n \geq 3$ vertices in turn implies the following.

Corollary 6.5. Let $U$ be the group of all unipotent upper triangular $d \times d$ matrices, where $d \geq 4$, and let $\Gamma$ be a lattice in $U$. Then $U / \Gamma$ does not admit any ergodic automorphism. In particular, it does not admit any Anosov automorphism.

Proof. We note that every lattice in $U$ is arithmetic (see [10]) and hence it suffices to prove the assertion for the lattice $\Gamma$ consisting of integral matrices in $U$. Let $N=U /[U,[U, U]]$. Through an inspection of the matrix representation it is easy to see that $N$ is then the simply connected nilpotent Lie group associated with the chain graph over $d-1$ vertices; this may be realised, for instance, by taking $S=\left\{E_{12}, \ldots, E_{(d-1) d}\right\}$ (in that order). Also, $[U,[U, U]] \Gamma$ is closed and $\Gamma^{\prime}=[U,[U, U]] \Gamma /[U,[U, U]]$ is a lattice in $N$, and it is the lattice associated with the graph. Therefore $N / \Gamma^{\prime}$ does not admit any ergodic automorphism. Since any ergodic automorphism of $U / \Gamma$ would factor to an ergodic automorphism of $N / \Gamma^{\prime}$, it follows that $U / \Gamma$ also does not admit any ergodic automorphism.

We note that the cycle graphs over $n \geq 5$ vertices are included in (i), the 3cycle which is a complete graph, is included in (ii), and the 4-cycle is also included in (ii), as it is a clump graph with $k=2$ and (i.e., a bipartite graph with) $S_{1}$ and $S_{2}$ consisting of the alternate vertices in the cycle.

In the case of the complete graphs, which includes also the cycle graph over 3 vertices, the existence of Anosov automorphisms was known earlier (see [1]). The other examples given in Corollary 6.4(ii) and (iii) are not found in literature. We note that the 4-cycle graph which is the simplest from these, gives an example of an 8-dimensional compact nilmanifold $N / \Gamma$ admitting an Anosov automorphism.

We note also the following. Let $(S, E)$ be a finite graph and let $(S, \tilde{E})$ be the complementary graph (see $\$ 5$ ). Let $N / \Gamma$ and $\tilde{N} / \tilde{\Gamma}$ be the compact nilmanifolds associated with $(S, E)$ and $(S, \tilde{E})$ respectively. Recall that the decomposition of $S$ into coherent components is the same with respect to $E$ and $\tilde{E}$. If each of the coherent components has at least three elements, then both $N / \Gamma$ and $\tilde{N} / \tilde{\Gamma}$ admit Anosov automorphisms. If there are singleton coherent components, then neither admits Anosov automorphisms. If there are no singleton coherent components but there are coherent components with two elements, existence of Anosov automorphisms will depend on the edge configuration and it may happen that one of them or neither may admit Anosov automorphisms. 
We shall now apply Corollary 6.4 to deduce Corollary 1.2. For this we need the following lemma.

Lemma 6.6. Let $(S, E)$ be a connected graph. Then the simply connected nilpotent Lie group $N$ associated with $(S, E)$ is indecomposable.

Proof. Let $N_{1}$ and $N_{2}$ be closed connected normal subgroups of $N$ such that $N_{1} \cap$ $N_{2}$ is trivial and $N=N_{1} N_{2}$. Without loss of generality, we may assume that $N_{1}$ is nontrivial. Let $\mathcal{N}$ be the Lie algebra corresponding to $N$, namely the Lie algebra associated with $(S, E)$, and let $\mathcal{N}_{1}$ and $\mathcal{N}_{2}$ be the Lie subalgebras of $\mathcal{N}$ corresponding to $N_{1}$ and $N_{2}$ respectively. Recall that $\mathcal{N}=V \oplus W$ (notation as in \$1) and let $V_{1}$ and $V_{2}$ be the projections of $\mathcal{N}_{1}$ and $\mathcal{N}_{2}$ respectively, on $V$. Since elements of $\mathcal{N}_{1}$ and $\mathcal{N}_{2}$ commute with each other and $W$ is contained in the center it follows that $\left[v_{1}, v_{2}\right]=0$ for all $v_{1} \in V_{1}$ and $v_{2} \in V_{2}$. Let $S_{1}$ (respectively $S_{2}$ ) be the subset consisting of all $\alpha$ in $S$ such that there exists $v$ in $V_{1}$ (respectively in $V_{2}$ ) such that $v=a \alpha+\sum_{\xi \in S, \xi \neq \alpha} a_{\xi} \xi$ with $a \neq 0$. Since $N_{1}$ is nontrivial, it follows that $S_{1}$ is nonempty. Suppose if possible that $S_{2} \backslash S_{1}$ is nonempty. As the graph is connected we can then find $\alpha \in S_{1}$ and $\beta \in S_{2} \backslash S_{1}$ such that $\alpha \beta \in E$. Hence there exist $v_{1} \in V_{1}$ and $v_{2} \in V_{2}$ such that $v_{1}=a_{1} \alpha+\sum_{\xi \in S_{1}, \xi \neq \alpha} a_{\xi} \xi$ and $v_{2}=a_{1} \beta+\sum_{\eta \in S_{2}, \eta \neq \beta} a_{\eta} \eta$, with $a_{1}$ and $a_{2}$ nonzero. Since $\beta \notin S_{1}$, it follows that $\left[v_{1}, v_{2}\right]=a_{1} a_{2}[\alpha, \beta]+w$, where $w$ is contained in the subspace of $W$ spanned by $\{[\xi, \eta]: \xi, \eta \in S, \xi \wedge \eta \neq \alpha \wedge \beta\}$. Since $a_{1} a_{2}$ is nonzero, this implies that $\left[v_{1}, v_{2}\right]$ is nonzero, which is a contradiction to the observation above. Therefore, $S_{2} \backslash S_{1}$ is empty, which means that $S_{2} \subset S_{1}$ and hence $S_{1}=S$. Thus for all $\alpha \in S$ there exists a $v_{\alpha} \in V_{1}$ whose $\alpha$-coordinate is nonzero. Since $S_{1}=S$, taking a suitable linear combination of such vectors, we get a $v \in V_{1}$ with $v=\sum_{\alpha \in S} a_{\alpha} \alpha$ with $a_{\alpha} \neq 0$ for all $\alpha \in S$. We shall deduce from this that $V_{1}=V$. Let $v^{\prime}=\sum_{\xi \in S} b_{\xi} \xi$ be any element of $V_{2}$. Then we have $\left[v, v^{\prime}\right]=0$, which implies that if $\alpha, \beta \in S$ and $\alpha \beta \in E$, then $a_{\alpha} b_{\beta}-a_{\beta} b_{\alpha}=0$, and hence $b_{\alpha} / a_{\alpha}=b_{\beta} / a_{\beta}$. Since the graph is connected, this shows that $b_{\alpha} / a_{\alpha}$ is the same for all $\alpha \in S$ which means that $v^{\prime}$ is a multiple of $v$, and, in particular, belongs to $V_{1}$. As this applies to every element $v^{\prime}$ of $V_{2}$, we get that $V_{2}$ is contained in $V_{1}$, and hence $V_{1}=V$, as sought to be shown. The conclusion means that $\mathcal{N}_{1}+W=V+W=\mathcal{N}$. Recall that $W=[\mathcal{N}, \mathcal{N}]$. Substituting for $\mathcal{N}$ as $\mathcal{N}_{1}+W$ from the last equation we get that $W=\left[\mathcal{N}_{1}+W, \mathcal{N}_{1}+W\right] \subset \mathcal{N}_{1}$, and hence $\mathcal{N}=\mathcal{N}_{1}+W=\mathcal{N}_{1}$. Therefore, $N_{1}=N ;$ this proves the lemma.

Proof of Corollary 1.2, For each finite graph $(S, E)$ let $d(S, E)$ denote the the sum of the cardinalities of $S$ and $E$. We note that $d(S, E)$ is the dimension of the simply connected Lie group associated with $(S, E)$. Let $D$ denote the set of integers $n$ such that there exists a connected graph $(S, E)$ such that $d(S, E)=n$, and the compact nilmanifold associated with it admits an Anosov automorphism. We show that all integers $n \geq 17$ are contained in $D$. In view of Lemma 6.6 this would imply the first assertion in the corollary. We shall also consider along the way the number $\nu(n)$ of compact nilmanifolds of dimension $n$ arising in this way, and prove the estimate as in the statement of the corollary.

Consider the following parametrised family of graphs. Let $S$ be a set which is a union of subsets $S_{1}, \ldots, S_{k}, S_{k+1}$, where $k \geq 1, S_{1}, \ldots, S_{k}$ consist of two elements each, and $S_{k+1}$ has $q \geq 2$ elements. Let $\mathcal{E}$ be a set of unordered pairs of elements from $\{1, \ldots, k, k+1\}$ such that the following conditions are satisfied: 
i) $(\{1, \ldots, k+1\}, \mathcal{E})$ is a connected graph, ii) the vertex $k+1$ is on only one edge. Consider the clump graph $(S, E)$ on $S=\bigcup_{i=1}^{k+1} S_{i}$ corresponding to the graph $(\{1, \ldots, k+1\}, \mathcal{E})$ (see $\$ 5$ item 5 , for definition). Let $p$ be the number of edges in $\mathcal{E}$ involving only the vertices $1, \ldots, k$. Clearly, the number of edges in $E$ is $4 p+2 q$. Hence $d(S, E)=2 k+3 q+4 p$. By Corollary 6.4 the compact nilmanifolds associated with clump graphs admit Anosov automorphisms, so we get that $2 k+3 q+4 p$ is contained in $D$; in this $k \geq 1$ and $q \geq 2$ can be chosen arbitrarily, and $p$, which is the number of edges of a connected graph on $\{1, \ldots, k\}$ ranges between $k-1$ and $\frac{1}{2}(k-1) k$. In particular, choosing $k=4$ we get that $\{3 q+4 p+8: 3 \leq p \leq 5, q \geq 2\}$ is contained in $D$. It can be verified that this set contains all integers $n \geq 32$.

Before going over to proving the first statement in the corollary for lower values of $n$, we shall deduce a lower bound on $\nu(n)$, using the above family of graphs. Let an integer $n$ be given. We choose $q=2$ if $n$ is even and $q=3$ if $n$ is odd. As $d(S, E)=2 k+3 q+4 p$, the condition for it to equal $n$ is $k+2 p=m$, where $m=(n-6) / 2$ if $n$ is even, and $(n-9) / 2$ if $n$ is odd. For any integer $k$ with the same parity as $m$ contained in the interval $\left[\sqrt{m}, \frac{1}{3}(m+2)\right]$ we get an integer $p$ such that $k+2 p=m$ and $k-1 \leq p \leq \frac{1}{2}(k-1) k$. For each of these values of $k$ we get a connected graph $(S, E)$ as above for which $d(S, E)=n$. We note also that for distinct graphs from this family the associated nilpotent Lie groups are nonisomorphic as the dimensions of their commutators are different. This shows that $\nu(n) \geq \frac{1}{2}\left(\frac{1}{3}(m+2)-\sqrt{m}-2\right) \geq \frac{1}{12}(n-3 \sqrt{2 n}-17)$ for all $n$. This proves the second assertion in the corollary.

To complete the proof of the first part we now produce examples showing that all integers from 17 to 31 , are contained in the set $D$ as above. In each of the examples below the existence of Anosov automorphisms follows from Corollary 6.4

First we note that choosing $k=3$ in the family of graphs introduced above, and taking $p$ to be 2 or 3 as is admissible, we get that $\{20,23,26,29\}$ and $\{24,27,30\}$ are contained in $D$. For $k=2$, we can choose $p=1$ and this gives that 17 also belongs to $D$.

Now let $S$ be a set consisting of 10 elements and $S_{1}, S_{2}$ and $S_{3}$ be mutually disjoint subsets consisting of 2,3 and 5 elements respectively. Let $(\{1,2,3\}, \mathcal{E})$ be the graph with edges connecting 1 with 2 and 2 with 3 . Consider the clump graph $(S, E)$ corresponding to the graph $(\{1,2,3\}, \mathcal{E})$. The number of edges is 21 . This shows that 31 is contained in $D$. The bipartite graph on a set $S$ of 7 elements partitioned into subsets of 3 and 4 elements yields that 19 belongs to $D$.

The complete graphs on sets of 6 and 7 vertices provide examples with $d(S, E)=$ 21 and 28 respectively, thus showing that 21 and 28 are contained in $D$.

Consider a magnet graph $(S, E)$ (see $\$ 5$ item 6 , for definition), with $C$ as the core subset. If $S$ has 7 elements and $C$ has 4 , then $d(S, E)=25$. If $S$ has 7 elements and $C$ has 3 elements, then $d(S, E)=22$, and if $S$ has 6 elements and $C$ has 3 elements, then $d(S, E)=18$. Thus 18,22 and 25 are also contained in $D$.

Together the above observations show that $D$ contains all integers $n \geq 17$. This completes the proof of the corollary.

Remark 6.7. It may also be noted that the lower bound for $\nu(n)$ obtained above is tentative and there is scope for improving it by considering other classes of graphs. Recall that in the proof mutual nonisomorphism of the Lie groups is ensured by the stronger condition of the dimensions of their commutators being different. In this respect we note that for a given $n$, the number of distinct $m$ for which there exists 
a nilpotent group $N$ associated to a connected graph with no singleton coherent component, with $N$ of dimension $n$ and $N /[N, N]$ of dimension $m$, is at most $\frac{1}{3}(n+2)$.

Remark 6.8. Let $D$ be the set of integers as in the proof of Corollary 1.2. The case with $k=1$ in the parametrised family of graphs, as in the proof, shows that 8,11 and 14 are contained in $D$. The complete graphs on 3,4 and 5 vertices yield, respectively that 6,10 and 15 are contained in $D$. It can be seen that the remaining values up to 16 do not belong to $D$; we omit the details of the verification.

We note that for some of the low values of dimensions not belonging to $D$ there could exist compact nilmanifolds $N / \Gamma$ (that are not associated with graphs) admitting Anosov automorphisms, with $N$ a 2-step simply connected nilpotent Lie group that is $\Gamma$-indecomposable (see $\S 1$ for definition), or even indecomposable, $\Gamma$ being a lattice in $N$. In principle this can be analysed using information on the structure of Lie groups and their representations in low dimensions; we shall, however, not go into it here, as the details turn out to be too cumbersome.

For dimensions 9 and 12 there exist compact nilmanifolds associated with connected graphs which admit ergodic automorphisms, though not Anosov automorphisms; these we get from magnet graphs over 4 and 5 vertices, with a core subset consisting of two elements in each case. And of course in dimension 3 the line graph on 2 vertices provides another such example; it is a quotient of the Heisenberg group.

\section{REFERENCES}

[1] L. Auslander and J. Scheuneman, On certain automorphisms of nilpotent Lie groups, Global Analysis (Proc. Sympos. Pure Math., Vol. XIV, Berkeley, Calif. 1968) pp. 9-15, Amer. Math. Soc., Providence, 1970. MR 42:5284

[2] A. Borel and J. Tits, Groupes Réductifs, Inst. Hautes Études Sci. Publ. Math. 27 (1965), 55-150. MR 34:7527

[3] S.G. Dani, Nilmanifolds with Anosov automorphisms, J. London Math. Soc. (2) 18 (1978), 553-559. MR 80k:58082

[4] S.G. Dani, On automorphism groups acting ergodically on connected locally compact groups, Ergodic Theory and Harmonic Analysis (Mumbai, 1999), Sankhya, Ser. A, 62 (2000), 360-366. MR 2001m:22013

[5] S.G. Dani, Some two-step and three-step nilpotent Lie groups with small automorphism groups, Trans. Amer. Math. Soc. 355 (2003), 1491-1503.

[6] K. Dekimpe, Hyperbolic automorphisms and Anosov diffeomorphisms on nilmanifolds, Trans. Amer. Math. Soc. 353 (2001), 2859-2877. MR 2002c:37043

[7] A. Manning, There are no new Anosov diffeomorphisms on tori, Amer. J. Math. 96 (1974), 422-429. MR 50:11324

[8] W. Parry, Ergodic properties of affine automorphisms and flows on nilmanifolds, Amer. J. Math. 91 (1969), 757-771. MR 41:5595

[9] G. Prasad and M.S. Raghunathan, Cartan subgroups and lattices in semisimple groups, Ann. Math. 90 (1972), 290-317. MR 46:1965

[10] M.S. Raghunathan, Discrete Subgroups of Lie Groups, Springer-Verlag, 1972. MR 58:22394a

[11] S. Smale, Differential dynamical systems, Bull. Amer. Math. Soc. 73 (1967), 747-817. MR 37:3598

School of Mathematics, Tata Institute of Fundamental Research, Homi Bhabha Road, Colaba, Bombay 400 005, India

E-mail address: dani@math.tifr.res.in

School of Mathematics, Tata Institute of Fundamental Research, Homi Bhabha Road, Colaba, Bombay 400005 , India

E-mail address: meera@math.tifr.res.in 